\title{
Comparison of the ganglion cell complex and retinal nerve fiber layer thickness in pseudoexfoliation syndrome, pseudoexfoliation glaucoma and healthy subjects
}

\author{
M Sinan Saricaoglu*, Ahmet Karakurt, Mualla Hamurcu and Selcan Ekicier Acar \\ Ankara Numune Training And Research Hospital, Eye Clinic, Ankara, Turkey
}

\begin{abstract}
Purpose: To evaluate the ganglion cell complex (GCC) and retinal nerve fiber layer (RNFL) thickness in the pseudoexfoliation syndrome (PEXS) and pseudoexfoliation glaucoma (PEXG) patients and comparison of the results with the healthy subjects.

Methods: In our study, 72 eyes of 45 patients were examined by the Cirrus HD-OCT after pupillary dilation. Nineteen eyes in PEXS (group 1), 23 eyes in PEXG (group 2) and 30 eyes in the control group (group 3) were included. The mean values of GCC, RNFL thickness measurements and quadrant measurement values (superior, inferior, nasal and temporal) were recorded. Results were compared statistically and statistical significance was accepted as $\mathrm{p}<0.05$.

Results: Central corneal thickness (CCT) mean values were $566.4 \pm 33 \mu$ in group $1,548.1 \pm 22.5 \mu$ in group 2 and $563.4 \pm 27.5 \mu$ in group 3 . Furthermore, no significant difference was detected in the CCT values (p>0.05). RNFL thickness measurements were $88.1 \pm 8.3 \mu$ in group 1 , 85.6 $\pm 10.9 \mu$ in group 2 and $100.7 \pm$ $11.9 \mu$ in group 3. Quadrant measurement values (superior, inferior, nasal and temporal) were respectively $110.2 \pm 15.4 \mu, 112.9 \pm 16.4 \mu, 71.8 \pm 10.6 \mu$ and $62.9 \pm 9.9 \mu$ in group $1,104.4 \pm 25.9 \mu, 113.6 \pm 15 \mu, 65.6 \pm 6.2 \mu, 59.9 \pm 9.5 \mu$ in group 2 and $122.8 \pm 18.1 \mu, 124.6 \pm 29.4 \mu, 78.9 \pm 25.7 \mu, 68.9 \pm 15.4 \mu$ in group 3 . The significant difference was detected in superior and nasal quadrant values of the RNFL thickness between the groups ( $<<0.05)$, no significant differences in the inferior and temporal quadrant values ( $p>0.05)$. PEX groups (group 1 and group 2 ) compared with the control group, the significant difference was detected in the mean and minimum GCC values $(\mathrm{p}<0.05)$.
\end{abstract}

Conclusions: The evaluation of the GCC and RNFL thickness measurements in the PEXS and PEXG patients can give valuable information to the clinician. Moreover, they can help to detect the transition from PEXS to PEXG.

\section{Introduction}

Pseudoexfoliation syndrome (PEXS) was first described in 1917 by Lindberg [1]. PEXS is an age-related systemic disease with primarily ocular manifestations characterized by deposition of whitish-gray pseudo exfoliation fibrillose-granular amyloid like material on the anterior lens capsule, zones, ciliary body, pupillary margin of the iris, corneal endothelium, anterior vitreous and trabecular meshwork $[2,3]$. The awareness of the significance of pseudoexfoliation has increased considerably in the latest decade. PEX is a risk factor not only for openangle glaucoma but also for angle-closure glaucoma, lens subluxation, blood aqueous barrier impairment serious intraoperative and postoperative complication and has been correlated with an increased incidence of cataract formation. Exfoliation of fibrillogranular amyloidlike material has been found in many organs such as skin, heart, lungs, liver, kidney, gall bladder, blood vessels, extraocular muscle, connective tissue in the orbit, optic nerves and meninges suggesting that PEXS is not only an ocular disease but also a general disorder that involves the abnormal production of extracellular matrix material $[4,5]$.

PEXS is recognized as an important risk factor for glaucoma. Pseudoexfoliation glaucoma (PEXG) is most often classified as a secondary glaucoma. Glaucoma is a progressive optic neuropathy that develops due to loss of retinal ganglion cells (RGC) and the retinal nerve fiber layer (RNFL) thickness comprising axons of these cells and is characterized by optic nerve damage. Glaucoma preferentially affects the three innermost retinal layers which are nerve fiber, ganglion cell and the inner plexiform layer. These three layers consist of axons, cell bodies and dendrites of ganglion cells respectively. The combination of these three layers is called the ganglion cell complex (GCC). The RGC population mostly exists in the macula. Developing methods capable of measuring glaucomatous changes associated with RGC loss allow glaucoma more accurately [6,7]. Among a variety of techniques, OCT has emerged to the forefront of ocular imaging which provides a wide variety of information. Macular thickness could be a valuable measure in evaluating glaucomatous structural changes in addition to assessment of the peripapillary RNFL mainly in primary open angle glaucoma and normotensive glaucoma [7-9].

The purpose of our study is to investigate the diagnostic value of the detection of RNFL and GCC thickness using spectral domain optical coherence tomography (SD-OCT) system in patients with PEXS, PEXG and normal group.

Correspondence to: M Sinan Saricaoglu, M.D, Associate Professor, Ankara Numune Training And Research Hospital, Eye Clinic, Ankara, Turkey, Tel: 090542749575; E-mail: msinansarica@yahoo.com

Key words: Pseudoexfoliation, glaucoma, retinal nerve fiber layer thickness, ganglion cell complex, optical coherence tomography

Received: August 08, 2017; Accepted: August 27, 2017; Published: August 30, 2017 
Saricaoglu MS (2017) Comparison of the ganglion cell complex and retinal nerve fiber layer thickness in pseudoexfoliation syndrome, pseudoexfoliation glaucoma and healthy subjects

\section{Material and methods}

In our study, 72 eyes of 45 patients were examined by the Cirrus HD-OCT after pupillary dilation. Nineteen eyes in PEXS (group 1), 23 eyes in PEXG (group 2) and 30 eyes in the control group (group 3) were included. The mean values of GCC, RNFL thickness measurements and quadrant measurement values (superior, inferior, nasal and temporal) were recorded. The patients consent forms were included. Study was organized according to the Declaration of Helsinki and Ankara Numune Education and Research Hospital ethics committee approval was received.

Group 1 included PEXS patients who have normal IOP (intraocular pressure) (under $21 \mathrm{mmHg}$ ), visible PEX material on the anterior segment structures, normal optic nerve head and normal visual field examination (Humprey 30-2, SITA-FAST). Group 2 included PEXG patients who have high IOP (over $21 \mathrm{mmHg}$ ), visible PEX material on the anterior segment structures, glaucomatous optic nerve head changes and visual field defects. Group 3 included healthy subjects who have normal IOP (under $21 \mathrm{mmHg}$ ), absence of PEX material on the anterior segment structures, healthy optic nerve head.

The exclusion criteria's were previous ocular surgery, ocular trauma, corneal pathology, posterior segment pathology, uveitis, medication usage that might influence anterior segment parameters, over one dioptre of cylindrical and/or three dioptres of spherical refractive errors and evident cataract.

Imaging was obtained using the Cirrus HD-OCT. Two scans, including one macular scan centered on the fovea (macular cube $200 \times 200$ protocol) and one peripapillary RNFL scan centered on the optic disc (optic disc cube $200 \times 200$ protocol), were acquired through dilated pupils by the same operator on the same day.

The ganglion cell analysis algorithm was included in the 6.0 software version of Cirrus HD-OCT. It detects and measures the thickness within a $6 \times 6 \times 2 \mathrm{~mm}$ elliptical annulus area centered on the fovea. The algorithm has been described previously in detail. In brief, the annulus has an inner vertical diameter of $1 \mathrm{~mm}$, which was chosen to exclude the portions of the fovea where the layers are very thin and difficult to detect accurately, and an outer vertical diameter of $4 \mathrm{~mm}$, which was chosen according to where the ganglion cell layer again becomes thin and difficult to detect.

The following GCC measurements were analyzed: average, minimum, for peripapillary RNFL thickness measurements, average thickness; superior, inferior, temporal, and nasal quadrant thickness; and RNFL thickness in the 12 clock hours vectors were included in the analysis. The software analyses the values, compares them to the device's internal normative database, and generates a thickness map, deviation map, and significance map color-coded to match RNFL thickness, with values within the normal range in green $(p=5 \%-95 \%)$, borderline values in yellow $(p=1 \%-5 \%)$, and values outside the normal range in red $(p<1 \%)$. Subjects with scans showing algorithm segmentation failure, signal strength $<6$, or artifacts due to eye movements or blinking were excluded from the study.

Statistical analysis was performed with SPSS for Windows version 13.0 (SPSS Inc, Chicago, IL, USA. The statistical significance was accepted as $\mathrm{p}<0.05$.

\section{Results}

Central corneal thickness (CCT) mean values were $566.4 \pm 33 \mu$ in group $1,548.1 \pm 22.5 \mu$ in group 2 and $563.4 \pm 27.5 \mu$ in group 3 .
Furthermore, no significant difference was detected in the CCT values $(\mathrm{p}>0.05)$.

Average RNFL thickness measurements were $88.1 \pm 8.3 \mu$ in group $1,85.6 \pm 10.9 \mu$ in group 2 and $100.7 \pm 11.9 \mu$ in group 3 . Quadrant measurement values (superior, inferior, nasal and temporal) were respectively $110.2 \pm 15.4 \mu, 112.9 \pm 16.4 \mu, 71.8 \pm 10.6 \mu$ and $62.9 \pm 9.9 \mu$ in group $1,104.4 \pm 25.9 \mu, 113.6 \pm 15 \mu, 65.6 \pm 6.2 \mu, 59.9 \pm 9.5 \mu$ in group 2 and $122.8 \pm 18.1 \mu, 124.6 \pm 29.4 \mu, 78.9 \pm 25.7 \mu, 68.9 \pm 15.4 \mu$ in group 3 (Table 1). The significant difference was detected in superior and nasal quadrant values of the RNFL thickness between the groups $(\mathrm{p}<0.05)$, no significant differences in the inferior and temporal quadrant values $(\mathrm{p}>0.05)$.

PEX groups (group 1 and group 2) compared with the control group. Mean and minimum GCC measurements were 79,57 $\pm 7,12$ and $74,84 \pm 14,07$ in group $1,75,09 \pm 12,01$ and $66,66 \pm 19,33$ in group $2,81,75 \pm 16,25$ and $71,84 \pm 23,57$ in control group. The significant difference was detected in the mean and minimum GCC values $(\mathrm{p}<$ 0.05) (Table 2).

\section{Discussion}

Glaucoma is the major cause of irreversible blindness in the world, in which the loss of the retinal ganglion cells is associated with clinically recognizable alterations in the retinal nerve fibers layer (RNFL) and optic nerve head $(\mathrm{ONH})$. Another retinal region which has been proposed to manifest changes in glaucoma is the macula (10). Because, more than $50 \%$ of the ganglion cells in the retina are located at macula and the ganglion cell layer is more than one cell layer thick at the macula $[10,11]$.

Identification of structural optic nerve damage is very important in the diagnosis of glaucoma and monitoring its clinical course. Until recently assessment of the optic nerve and RNFL has been subjective. New technologies have provided automated quantitative, reproducible and objective measurements of the optic nerve head and RNFL thickness. The OCT is one of these technologies capable of imaging, analyzing and documenting the optic disc head and RNFL morphology. In this regard, it has been proved by Li et al. [12] that RTVue-OCT may provide objective, quantitative and reproducible images of the optic nerve head and RNFL thickness in glaucoma. In our recent study, Saricaoglu et al. [13], compared OHT and POAG patients with normal population. They reported that in POAG patients there were statistically significant thinner RNFL thickness in all quadrants except temporal quadrant. Puska et al. [14] reported that during the follow-up

Table 1. RNFL thickness of the groups and statisticall analysis.

\begin{tabular}{|c|c|c|c|c|}
\hline RNFL $(\boldsymbol{\mu})$ & PEXS & PEXG & Control & $\mathbf{p}^{*}$ Value \\
\hline & Mean $( \pm \mathrm{SD})$ & Mean $( \pm \mathrm{SD})$ & Mean $( \pm \mathrm{SD})$ & \\
\hline Average & $88.1 \pm 8.3$ & $85.6 \pm 10.9$ & $100.7 \pm 11.9$ & $\mathrm{p}<0.05$ \\
\hline Superior & $110.2 \pm 15.4$ & $104.4 \pm 25.9$ & $122.8 \pm 18.1$ & $\mathrm{p}<0.05$ \\
\hline Inferior & $112.9 \pm 16.4$ & $113.6 \pm 15$ & $124.6 \pm 29.4$ & $\mathrm{p}>0.05$ \\
\hline Nasal & $71.8 \pm 10.6$ & $65.6 \pm 6.2$ & $78.9 \pm 25.7$ & $\mathrm{p}<0.05$ \\
\hline Temporal & $62.9 \pm 9.9$ & $59.9 \pm 9.5$ & $68.9 \pm 15.4$ & $\mathrm{p}>0.05$ \\
\hline
\end{tabular}

"Statistical analysis was performed by Kruskal Wallis Test.

Table 2. Ganglion cell complex measurements of the groups and statisticall analysis.

\begin{tabular}{|c|c|c|c|c|}
\hline GCC $(\boldsymbol{\mu})$ & PEXS & PEXG & Control & $\mathbf{p}^{*}$ Value \\
\hline & Mean $( \pm \mathrm{SD})$ & Mean $( \pm \mathrm{SD})$ & Mean $( \pm \mathrm{SD})$ & \\
\hline Mean & $79,57 \pm 7,12$ & $75,09 \pm 12,01$ & $81,75 \pm 16,25$ & $\mathrm{p}<0.05$ \\
\hline Minimum & $74,84 \pm 14,07$ & $66,66 \pm 19,33$ & $71,84 \pm 23,57$ & $\mathrm{p}<0.05$ \\
\hline
\end{tabular}

"Statistical analysis was performed by Kruskal Wallis Test. 
Saricaoglu MS (2017) Comparison of the ganglion cell complex and retinal nerve fiber layer thickness in pseudoexfoliation syndrome, pseudoexfoliation glaucoma and healthy subjects

period, the IOP increased in the exfoliative and non-exfoliative eyes, and changes indicative of nerve fiber loss were measured in both eyes. In those in whom the IOP in the two eyes was equal throughout the follow-up period, disc changes took place only in the exfoliative eye [14]. Kim et al. [15] reported that macular GCC thickness and RNFL thickness showed similar diagnostic performance for to detecting early glaucoma.

In our study, the significant difference was detected in superior and nasal quadrant values of the RNFL thickness between the groups $(\mathrm{p}<0.05)$, no significant differences in the inferior and temporal quadrant values $(\mathrm{p}>0.05)$. Also, average RNFL thickness was lower in the PEXS and PEXG groups, it was statistically significant $(\mathrm{p}<0.05)$.

Previous technologies in glaucoma predominantly focused on the RNFL and ONH to evaluate the structural damage in glaucoma. There has been a renewed interest in scanning the macular region for detection of glaucomatous changes with the spectral domain optical coherence tomography [16]. Because, more than $50 \%$ of the ganglion cells in the retina are located at macula and the ganglion cell layer is more than one cell layer thick at the macula [16,17]. Macular scanning on SD-OCT evaluates the thickness of the inner layers of the retina that comprise the ganglion cell bodies and their appendages. Recent studies have been reported that the macular thickness parameters of SD-OCT were as good as the RNFL parameters in diagnosis of glaucoma [9,17-21].

In glaucomatous eyes, reduction in macular thickness is more significant, and loss of GCC is the main reason. Leung et al. [22] revealed that macular nerve fiber layer thickness decreases in glaucoma with respect to control subjects. The decrease in macular thickness was highly consistent with the changes in the structure of nerve fiber layer and visual function was reported by Greenfield et al. [23]. In that study, there is a strong relationship between the mean macular thickness and the mean deviation in the visual field, and conceived that diminution in macular thickness could be an indicator of the ganglion cell loss. Recent study Saricaoglu et al. [24] showed that macular thicknesses were significantly different between ocular hypertension and primary open angle glaucoma. Another study by Tan et al. showed that when nerve fiber layer values are evaluated together with macular GCC values, the diagnostic value increases from $78 \%$ to $87 \%$ in perimetric glaucoma and $45 \%$ to $56 \%$ in preperimetric glaucoma [8]. The macula region contains over $50 \%$ of all retinal ganglion cells and is likely an ideal region to detect early cell loss and changes over time because of the high density of cells [25]. In recent studies showed that, macular ganglion cell complex and ganglion cell layer with inner plexiform layer measurements not macular nerve fiber layer measurements were similar with circumpapillary retinal nerve fiber layer to diagnose preperimetric glaucoma [26].

In our study, PEX groups (group 1 and group 2) compared with the control group, the significant difference was detected in the mean GCC values. More recent studies also reported that close monitoring of nerve fiber layer thickness may facilitate early identification of those PEX eyes that convert to exfoliative glaucoma [27-30].

The first study about this subject was that Eltutar et al. [31] compared pseudoexfoliation syndrome with control subjects. Macular NFL, ganglion cell layer and inner plexiform layer, total peripapillary nerve fiber layer and ONH parameters were compared. They showed that inferior, temporal, nasal and total peripapillary nerve fiber layer thickness thinner than control subject. In our study we found that average, superior and nasal RNFL thickness thinner than control group. In that study they reported that the superior and total macular nerve fiber layer thickness, superior total GCC and inner plexiform layer thickness, superior, inner and total GCC thickness were thinner than control subjects [31]. In our study, we just compared mean and minimum GCC thickness and we detected significant difference in these GCC values. The difference of our study is that this the first report which compare PEXS and PEXG with normal group.

RNFL and GCC thickness of the PEXG was lower than PEXS (except inferior quadrant of RNLF thickness) but no significant differences was detected $(p>0.05)$. The evaluation of the GCC and RNFL thickness measurements in the pseudoexfoliation syndrome and glaucoma patients can give valuable information to the clinician. Moreover, they can help to detect transition from pseudoexfoliation syndrome to pseudoexfoliation glaucoma.

\section{References}

1. Linderg JG (1989) Clinical investigations on depigmentation of the pupillary border and translucency of the iris in cases of senile cataract and in normal eyes in elderly persons. Acta Ophthalmol 190: 1-96. [Crossref]

2. Schlötzer-Schrehardt U1, Naumann GO (2006) Ocular and systemic pseudoexfoliation syndrome. Am J Ophthalmol 141: 921-937. [Crossref]

3. Naumann GO, Schlötzer SU, Küchle M (1998) Pseudoexfoliation syndrome for the comprehensive ophthalmologist intraocular and systemic manifestations. Ophthalmology 105: 951-968. [Crossref]

4. Streeten BW, Li ZY, Wallace RN (1992) Pseudoexfoliative fibrillopathy in visceral organs of a patient with pseudoexfoliation syndrome. Arch Ophthalmol 110: 1757. 1762. [Crossref]

5. Shrum KR, Hattenhauer MG, Hodge D (2000) Cardiovascular and cerebrovascular mortality associated with ocular pseudoexfoliation. Am J Ophthalmol 129: 83-86. [Crossref]

6. Harwerth RS, Carter DL, Shen F, Smith EL, Crawford ML (1999) Ganglion cell losses underlying visual field defects from experimental glaucoma. Invest Ophthalmol Vis Sci 40: 2242-2250. [Crossref]

7. Sevim MS, Buttanri B, Acar BT, Kahya A, Vural ET, et al. (2013) Ability of fourierdomain optical coherence tomography to detect retinal ganglion cell complex atrophy in glaucoma patients. $J$ Glaucoma 22: 542-549. [Crossref]

8. Tan O, Chopra V, Lu AT, Schuman JS, Ishikawa H, et al. (2009) Detection of macula ganglion cellloss in glaucoma by Fourier-domain optical coherence tomography. Ophthalmology 116: 2305-2314. [Crossref]

9. Seong M, Sung KR, Choi EH, Kang SY, Cho JW, et al. (2010) Macular and peripapillary retinal nerve fiber layer measurements by spectral domain optical coherence tomography in normal-tension glaucoma. Invest Ophthalmol Vis Sci 51: 1446-1452. [Crossref]

10. Zeimer R, Asrani S, Zou S, Quigley H, Jampel H (1998) Quantitative detection of glaucomatous damage at the posterior pole by retinal thickness mapping. A pilot study. Ophthalmology 105: 224-231. [Crossref]

11. Curcio CA, Allen KA (1990) Topography of ganglion cells in human retina. J Comp Neurol 300: 5-25. [Crossref]

12. Li S, Wang X, Li S, Wu G, Wang N (2010) Evaluation of optic nevre head and retinal nerve fiber layer in early and advance glaucoma using frequency-domain optical coherence tomography. Graefes Arch Clin Exp Ophthalmol 248: 429-434. [Crossref]

13. Saricaoglu MS, Misir R, Karakurt A, Hasiripi H (2011) The Analysis of retinal nerve fiber layer thickness in patients with ocular hypertension and open angle glaucoma Glo-Kat 6: 92-96.

14. Puska P, Vesti E, Tomita G, Ishida K, Raitta C (1999) Optic disc changes in normotensive persons with unilateral exfoliation syndrome: a 3-year follow-up study. Graefes Arch Clin Exp Ophthalmol 237: 457-462. [Crossref]

15. Kim JS, Ishikawa H, Gabriele ML, Wollstein G, Bilonick AR, et al. (2010) Retina nerve fiber layer thickness measurement comparability between time domain optical coherence tomography (OCT) and spectral domain OCT. Invest Ophthalmol Vis Sci 51: 896-902. [Crossref]

16. Begum VU, Jonnadula GB, Yadav RK, Addepalli UK, Senthil S, et al. (2014) Scanning the macula for detecting glaucoma. Indian J Ophthalmol 62: 82-87. [Crossref] 
Saricaoglu MS (2017) Comparison of the ganglion cell complex and retinal nerve fiber layer thickness in pseudoexfoliation syndrome, pseudoexfoliation glaucoma and healthy subjects

17. Rao HL, Babu JG, Addepalli UK, Senthil S, Garudadri CS (2012) Retinal nerve fiber layer and macular inner retina measurements by spectral domain optical coherence tomograph in Indian eyes with early glaucoma. Eye 26: 133-139. [Crossref]

18. Rao HL, Leite MT, Weinreb RN, Zangwill LM, Alencar LM, et al. (2011) Effect of disease severity and optic disc size on diagnostic accuracy of RTVue spectral domain optical coherence tomograph in glaucoma. Invest Ophthalmol Vis Sci 52: 1290-1296. [Crossref]

19. Garas A, Vargha P, Holló G (2011) Diagnostic accuracy of nerve fibre layer, macular thickness and optic disc measurements made with the RTVue-100 optical coherence tomograph to detect glaucoma. Eye (Lond) 25: 57-65. [Crossref]

20. Schulze A, Lamparter J, Pfeiffer N, Berisha F, Schmidtmann I, et al. (2011) Diagnostic ability of retinal ganglion cell complex, retinal nerve fiber layer, and optic nevre head measurements by Fourier-domain optical coherence tomography. Graefes Arch Clin Exp Ophthalmol 249: 1039-1045. [Crossref]

21. Carbonelli SG, Piero MB (2011) Spectral-domain optical coherence tomography forthe diagnosis and follow-up of glaucoma. Curr Opin Ophthalmol 22: 115-123. [Crossref]

22. Leung CK, Chan WM, Yung WH, Ng AC, Woo J, et al. (2005) Comparison of macular and peripapillary measurements for the detection of glaucoma: an optical coherence tomography study. Ophthalmology 112: 391-400. [Crossref]

23. Greenfield DS, Bagga H, Knighton RW (2003) Macular thickness changes in glaucomatous optic neuropathy detected using optical coherence tomography. Arch Ophthalmol 121: 41-46. [Crossref]
24. Saricaoglu MS, Misir R, Karakurt A (2011) The analysis of macular thickness measurements in patients with ocular hypertension and primary open angle glaucoma. Glo-Kat 6: 250-253.

25. Wollstein G, Schuman JS, Price LL, Aydin A, Beaton SA, et al. (2004) Optical coherence tomography (OCT) macular and peripapillary retinal nerve fiber layer measurements and automated visual fields. Am J Ophthalmol 138: 218-225. [Crossref]

26. Kim YJ, Kang MH, Cho HY, Lim HW, Seong M (2014) Comparative study of macular ganglion cell complex thickness measured by spectral-domain optical coherence tomography in healt eyes, eyes with preperimetric glaucoma, and eyes with early glaucoma. Jpn J Ophthalmol 58: 244-251. [Crossref]

27. Dimopoulos AT, Katsanos A, Mikropoulos DG, Giannopoulos T, Empeslidis T, et al (2013) Scanning laser polarimetry in eyes with exfoliation syndrome. Eur J Ophthalmol 23: 743-750. [Crossref]

28. Kozobolis VP, Glynatsis M, Labiris G, Katsanos A, Fanariotis M, et al. (2010) Retinal nerve fiber layer thickness in patients with exfoliation, exfoliative glaucoma, and primary open angle glaucoma. Eur J Ophthalmol 20: 142-148. [Crossref]

29. Mohamed MM (2009) Detection of early glaucomatous damage in pseudoexfoliation syndrome by assessment of retinal nerve fiber layer thickness. Middle East Afr $J$ Ophthalmol 16: 141-145. [Crossref]

30. Rao A (2012) Clinical and optical coherence tomography features in unilateral versus bilateral pseudoexfoliation syndrome. J Ophthalmic Vis Res 7: 197-202. [Crossref]

31. Eltutar K, Acar F, Öztürker ZK, Unsal E, Erkul SÖ (2015) Structural Changes in Pseudoexfoliation Syndrome Evaluated with Spectral Domain Optical Coherence Tomography. Curr Eye Res 6:1-8. [Crossref]

Copyright: $\mathbb{C} 2017$ Saricaoglu MS. This is an open-access article distributed under the terms of the Creative Commons Attribution License, which permits unrestricted use, distribution, and reproduction in any medium, provided the original author and source are credited. 